Research Paper

\title{
Treatment of Neuroblastoma with an Engineered "Obligate" Anaerobic Salmonella typhimurium Strain YB1
}

\author{
Bo-Tao Ning1, ${ }^{*}$, Bin $\mathrm{Yu}^{3^{*}}$, Shing Chan², Jian-liang Chan², Jian-Dong Huang ${ }^{3 凶}$, Godfrey Chi-Fung Chan ${ }^{2,4 \bowtie}$ \\ 1. Pediatric Intensive Care Unit, Shanghai Children's medical Center affiliated to Shanghai Jiao Tong University School of Medicine, Shanghai, PR China; \\ 2. Department of Peadiatrics \& Adolescent, Queen Mary Hospital, LKS Faculty of Medicine, the University of Hong Kong, Pokfulam, Hong Kong SAR, PR \\ China; \\ 3. Department of Biochemistry, LKS Faculty of Medicine, the University of Hong Kong, Pokfulam, Hong Kong SAR, PR China; \\ 4. Center of Cancer Research, LKS Faculty of Medicine, the University of Hong Kong, Pokfulam, Hong Kong SAR, PR China. \\ * Equal contribution \\ $\bowtie$ Corresponding authors: J.D.H. (jdhuang@hku.hk) or G.C.F.C. (gcfchan@hkucc.hku.hk)
}

(C) Ivyspring International Publisher. This is an open access article distributed under the terms of the Creative Commons Attribution (CC BY-NC) license (https://creativecommons.org/licenses/by-nc/4.0/). See http://ivyspring.com/terms for full terms and conditions.

Received: 2016.12.27; Accepted: 2017.02.27; Published: 2017.06.03

\begin{abstract}
Purpose Neuroblastoma is an embryonic solid tumor derived from the progenitors of the sympathetic nervous system. More than half of the patients developed metastatic disease at the time of initial diagnosis and had poor outcome with current therapeutic approaches. In recent years, some obligate and facultative anaerobic bacteria were reported to target the hypoxic and necrotic region of solid tumor models and caused tumor regression. We recently successfully constructed an "obligate" anaerobic Salmonella strain YBI that was applied in breast cancer nude mice model by us. Here, we report the application of YBI in neuroblastoma treatment.

Methods The anti-cancer effect and side-effects of YBI was examined in both in vitro and in vivo experiment. Previous established orthotopic neuroblastoma SCID/beige murine model using SK-NLP/luciferase cell line was adopted.

Results In vitro, YB1 induced apoptosis for up to $31.4 \%$ of the neuroblastoma cells under anaerobic condition, three times more than that under aerobic condition (10.9\%). The expression of both Toll like Receptor 4 and 5 (TLR4 and TLR5) in cancer cells were significantly up-regulated $(p<0.05, p<0.01$ respectively) after the treatment of $Y B 1$ under anaerobic condition. In mouse model, $Y B 1$ preferentially accumulated inside the core of the tumors, rather than in normal tissues as our previous reported. This is suggestive of the hypoxic nature of tumor core. Tumor growth was significantly retarded in YBI treatment group $(n=6, P<0.0 I)$. Furthermore, there was no long-term organ damage noted in all the organs examined including heart, lung, liver, spleen and brain in the YB1 treated mice.

Conclusion The genetic modified Salmonella strain YBI is a promising anti-tumor strategy against the tumor bulk for neuroblastoma. Future study can be extended to other common cancer types to verify the relative efficacy on different neoplastic cells.
\end{abstract}

Key words: neuroblastoma, xenograft, Salmonella, hypoxia, target-killing.

\section{Introduction}

Neuroblastoma is one of the most common extra-cranial solid tumors found in infancy and early childhood, which is diagnosed at a median age of about 17 months. It is known that early and advanced stage neuroblastoma behave differently and they are two biologically different types of tumors. However, majority of the neuroblastoma found after 18-month-old belongs to the advanced stage with metastasis. They have very poor prognosis even with current multi-modality therapeutic approach. Neuroblastoma can arise from anywhere along the sympathetic nervous system, but majority of them have the primary tumor in the adrenal medulla [1]. To simulate such natural microenvironment, intra-adrenal orthotopic xenograft neuroblastoma mouse model is a valuable tool in improving our 
understanding and control of neuroblastoma. It allows tumor growth and metastasis to take place and makes it convenient, precise and reliable for drugs experiment in vivo [2,3]. Research findings suggested that neuroblastoma cancer stem cells and the cancer niche are thriving better in hypoxic microenvironment and they are resistant to most treatments. It is because the therapeutic agents or cytotoxic drugs cannot penetrate the poorly vascularized, hypoxic regions of the tumors, therefore leaving these sites untreated $[4,5]$.

This prompted researchers to search for an alternative treatment strategy. An ideal tumor therapy vector should be specifically present in tumor hypoxic regions, while absent in normal tissues. This kind of vector may help to target the most resistant area of the tumor. Fortunately, facultative anaerobes, such as Salmonella typhimurium, can target both small and large tumors and reduce tumor size [6]. While wild type Salmonella strains may shrink tumors, their natural pathological virulence may result in the death of the host [7]. We recently engineered an "obligate" anaerobic Salmonella strain YB1 [8]. This strain was derived from a vaccine strain SL7207. It been reported to be an anticancer agent [9-13]. However, it is lethal if injected intravenously even though SL7207 is aroA defective [14]. In YB1, the essential gene asd has been genetically engineered to be controlled by hypoxia conditioned promoter. The asd gene is responsible for the synthesis of diaminopimelic acid (DAP), which is an essential component of the cell wall in gram-negative bacteria. Deficiency of DAP will lead to lysis of the bacterium, unless extrinsic DAP is supplied by the environment. The YB1 can only survive under hypoxic condition (less than 0.5\% oxygen) without additional DAP [8]. The normal functions of this bacterium are not compromised by the deletion or mutation of any of its genes. The anti-tumor effect of YB1 in nude has been tested in mice breast tumor and neuroblastoma model by our group [8, 15]. In normal tissues under aerobic conditions, since asd is not expressed, YB1 are rapidly eliminated. In tumor, we further found that YB1 accumulated inside hypoxic region and caused tumor regression [8].

In this study, we attempt to investigate the tumor-targeting and potential tumor-repression effect of Salmonella YB1 against neuroblastoma. The possible association of apoptosis and TLRs genes expression was determined. The potential toxic effect to the normal tissues induced by either the genetic modified YB1 or the original Salmonella strain SL7207 on orthotopic SCID/beige mice bearing neruroblastoma was evaluated.

\section{Materials and Methods}

\section{Bacterial strains, animals, cell lines, reagents, primers and chemicals}

Salmonella typhimurium strain SL7207 was kindly provided by Dr. Stocker [14] . The culture of SL7207 and YB1 was described previously [8]. Six-week-old male SCID/beige mice were purchased from the Laboratory Animal Unit of The University of Hong Kong. The research protocols were approved by the Committee on the Use of Live Animals in Teaching and Research of the University of Hong Kong (CULATR 2360-11) with the related animal license issued by the local Department of Health. The gene transfected neuroblastoma cell line SK-NLP/luciferase were cultured in Dulbecco's modified Eagle medium (DMEM; Invitrogen, Carlsbad, CA), containing 1000 $\mu \mathrm{g} / \mathrm{ml}$ G418 (Roche, Mannheim, Germany), 10\% fetal bovine serum (FBS; Hyclone, Logan, UT), $100 \mathrm{U} / \mathrm{mL}$ penicillin, 100 $\mathrm{mg} / \mathrm{mL}$ streptomycin, and $2 \mathrm{mM}$ L-glutamine (Invitrogen, New York, USA). Cultures were maintained at $37^{\circ} \mathrm{C}$ in a humidified atmosphere containing 5\% $\mathrm{CO}_{2}$. Luciferin, $0.05 \%$ trypsin with $25 \mathrm{mM}$ EDTA, Superscript II reverse transcriptase and DNase I were all bought from Invitrogen (New York, USA). Matrix and Annexin V were purchased from BD company (San Diego, USA). SYBR ${ }^{\circledR}$ Green PCR Master Mix kit was bought from Applied Biosystems (Warrington, UK). AllPrep ${ }^{\circledR}$ RNA Mini kit was purchased from QIAGEN (Hilden, Germany). Primers were synthesized by Integrated DNA Technologies (Iowa, USA). Rabbit anti-Salmonella antibody, mouse anti-TLR4 and 5 monoclonal antibodies and horseradish peroxidase conjugated secondary antibody were from Abcam (Cambridge, UK). DAB solution was from DACO (Tokyo, Japan). Anaerobic jar was from Mitsubishi Gas Chemical Company (Tokyo, Japan). The 3600M021-Metabolic cages were bought from Tecniplast Company (Buguggiate, Italy). Meloxicam was bought from Boehringer Ingelheim (Auckland, New Zealand). Chemicals were from Sigma (St. Louis, USA). Antibiotic stocking solutions were prepared as follows: Chloramphenicol, $25 \mathrm{mg} / \mathrm{ml}$ in methanol; Ampicillin, 100 mg/ml; Gentamycin, 50 mg/ml. Strains culture were supplied with $50 \mu \mathrm{g} / \mathrm{ml}$ DAP where noted.

\section{Salmonella invasion of neuroblastoma cells in vitro}

Salmonella and SK-NLP/luciferase cells were prepared and co-cultured at a ratio of 500:1 for 2 hours under anaerobic or aerobic conditions. The cells 
were then washed with PBS and cultured in gentamycin supplemented medium for 20 minutes to kill extracellular bacteria, then washed with PBS and added the medium with $100 \mu \mathrm{g} / \mathrm{ml}$ gentamycin to kill the residual bacteria. 24 hours later, tumor cells apoptosis induced by bacteria under aerobic and anaerobic conditions were detected by annexin $\mathrm{V}$ assay according to manufacturer's instructions through flow cytometry.

Total cellular RNA was extracted from cultured cells using AllPrep ${ }^{\circledR}$ RNA Mini kit according to the manufacturer's instructions. Reverse transcription was performed on the DNase I-treated RNA using Superscript II reverse transcriptase according to the manufacturer's recommendation. The cDNA that was synthesized was subjected to PCR amplification, and the PCR product was quantified by real-time PCR using the SYBR ${ }^{\circledR}$ Green PCR Master Mix. The primers that were used are shown in Table 1 . The $\beta$-actin gene was amplified as an internal control.

Table 1. DNA sequences of primers used in quantitative PCR analysis

\begin{tabular}{ll}
\hline Gene & Sequence \\
\hline 3-actin & F: 5'-CCGTCTTCCCCTCCATCGT-3' \\
& R: 5'-ACTTCAGGGTGAGGATGCC-3' \\
TLR4 & F:5'-AGAGTTTCCTGCAATGGATCAAG-3' \\
& R: 5'-TTATCTGAAGGTGTTGCACATTCC-3' \\
TLR5 & F: 5'-GGCTTAATCACACCAATGTCACTATAG-3' \\
& R: 5'- TTAAGACTTCCTCTTCATCACAACCTT-3' \\
\hline
\end{tabular}

$F$, forward primer; $R$, reverse primer.

\section{Establishment of orthotopic neuroblastoma SCID/beige murine model [15]}

6-week SCID/beige male mice were inoculated with $0.2 \times 10^{6} \quad$ SK-NLP/luciferase cells through intra-adrenal injection under the conditions of surgical microscope and anesthesia. Animals were anesthetized by intra-peritoneal administration of 100 $\mathrm{mg} / \mathrm{kg}$ pentobarbital. Subsequently, fur on the left side of the body between the fore and hind legs was clipped and prepared with alcohol and betadine. An incision was made over the left retro-peritoneal space to expose the left adrenal gland, and $10 \mu \mathrm{l}$ matrix containing $0.2 \times 10^{6}$ neuroblastoma cells were injected into the gland with a disposable $29 \mathrm{G}$ needle attached to a $300 \mu \mathrm{l}$ syringe. The incision was sutured with 5-0 dexon. The animals were allowed to completely recover from anesthesia before being returned to the cage. After surgery, the meloxicam in drinking water was administered to minimize the pain at a dose of $0.3 \mathrm{mg} / \mathrm{kg}$, 24 hourly for 3 days. Two weeks after the surgery, the tumor development was monitored under In Vivo Imaging System (IVIS, Xenogen IVIS 100).
Target neuroblastoma with Salmonella YB1 in orthotopic murine model and side-effects observation

To measure the targeting and therapeutic effect of bacterial inoculation on orthotopic neuroblastoma murine model, three groups of mice $(n=6)$ were treated with either YB1 $5 \times 10^{7}$ Colony Forming Units (CFU) /mouse, SL7207 $5 \times 10^{7} \mathrm{CFU} /$ mouse or PBS with volume of $100 \mu \mathrm{l}$ injected through the tail vein. Tumor growth was detected by Xenogen IVIS 100 two weeks after the treatment. Survival rate and body weight were recorded every 2 days. To measure the bacterial distribution after tail vein injection, mice were sacrificed and tissues were weighed, homogenized, serially diluted in PBS and plated with the chloramphenicol and DAP. CFU were counted after two days' culture. To observe the side-effect, YB1 $\left(5 \times 10^{7} \mathrm{CFU} /\right.$ mouse) and SL7207 $\left(5 \times 10^{7} \mathrm{CFU} /\right.$ mouse $)$ were also injected through tail vein to 6 SCID health mice, respectively. Organs were harvested and feces and urine were collected by metabolic cages. The presence of bacteria in different organs was determined.

\section{Immunohistochemical analysis}

Bacteria and PBS treated tumor bearing mice were injected with overdose pentobarbital for euthanasia. Tissues were removed from these mice and immediately fixed in $4 \%$ paraformaldehyde, paraffin embedded and sectioned. Salmonella was detected by rabbit anti-Salmonella antibody. TLR4 and 5 was detected by mouse anti-TLR4 and 5 monoclonal antibodies. Bound primary antibody was detected using horseradish peroxidase conjugated secondary antibody which then developed in DAB solution. Pictures were taken under a light microscope.

\section{Statistical analysis}

The unpaired, two-tailed Student $t$ test was used to determine differences between groups for the comparisons of Quantitative-PCR, tumor weight and CFU counts of salmonella YB1 using SPSS computer software (SPSS). Any $P$-value less than 0.05 is regarded as statistically significant.

\section{Results}

\section{Invasion of neuroblastoma cells by $\mathrm{YB} 1$ in vitro}

Neuroblastoma cell line SK-NLP/luciferase samples were co-cultured with YB1 or SL7207 under anaerobic or aerobic conditions. After removal of extracellular bacteria and overnight culture, the apoptotic rate of SK-NLP/luciferase cells in anaerobic conditions treated with each of the bacteria was assessed by annexin $\mathrm{V}$ assay. A significant increase in 
the number of apoptotic cells was noted in both YB1 $(31.4 \%)$ and SL7207 treated groups $(29.1 \%)$ when compared to negative control (1.02\%) under anaerobic condition. However, under aerobic conditions, the YB1 showed much less cytotoxic effect to the cancer cells $(10.8 \%)$, as compared to the $27.2 \%$ apoptosis of SL7207 treated cells (Fig. 1.), this result is consistent with our previous report in breast cancer model [8].

\section{Accumulation of YB1 in tumor and normal tissues in vivo}

The YB1 distribution in tumor and normal tissues were determined 2 weeks after treatment. In

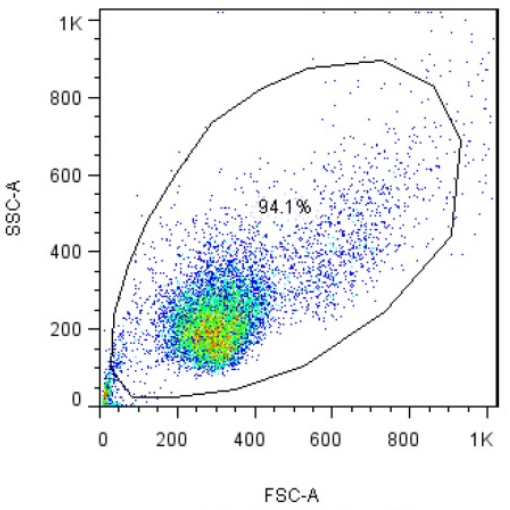

negative control

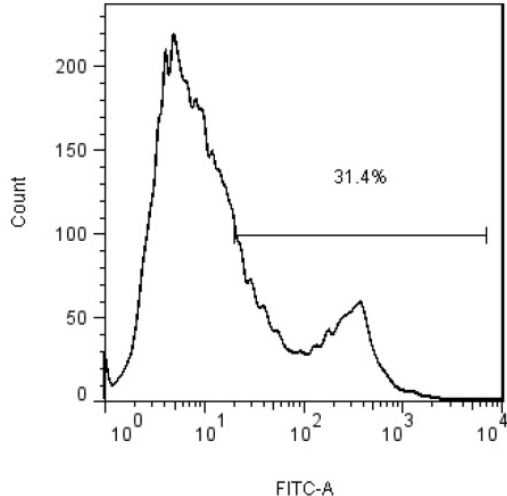

YB1 under anaerobic

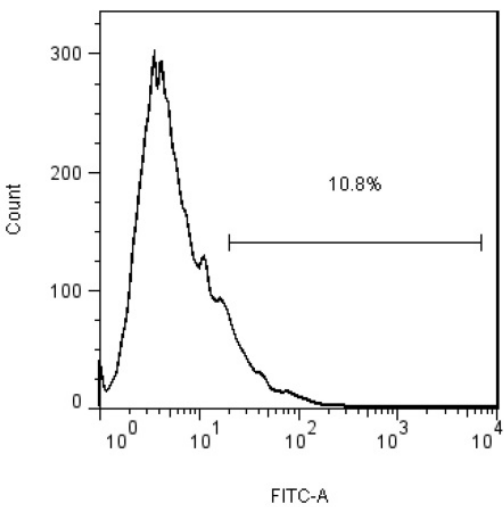

YB1 under aerobic tumors, YB1 was found to present in tumor with about $1.14 \times 10^{8} \mathrm{CFU} /$ gram. In hearts, spleens and livers, YB1 was found to be $6.38 \times 10^{4}, 9.81 \times 10^{3}$ and $3.73 \times 10^{3} \mathrm{CFU} /$ gram respectively (Fig. $2 A$ ). No bacteria were found in brain, lung, kidney, or blood. Immunohistochemical staining of sections of tumor, heart, liver and spleen confirmed the distribution of Salmonella bacteria in these tissues (Fig. 2B). There was some parenchymal infiltration in the liver but the distribution in the tumor was more diffused and extensive.

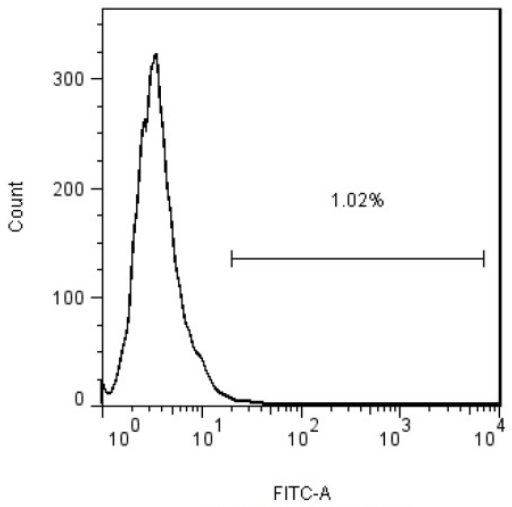

negative control

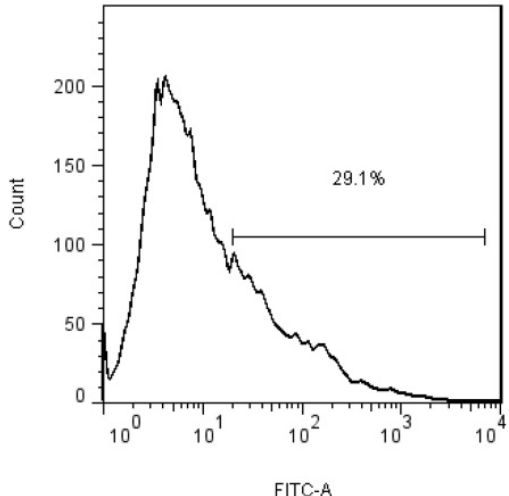

SL7207 under anaerobic

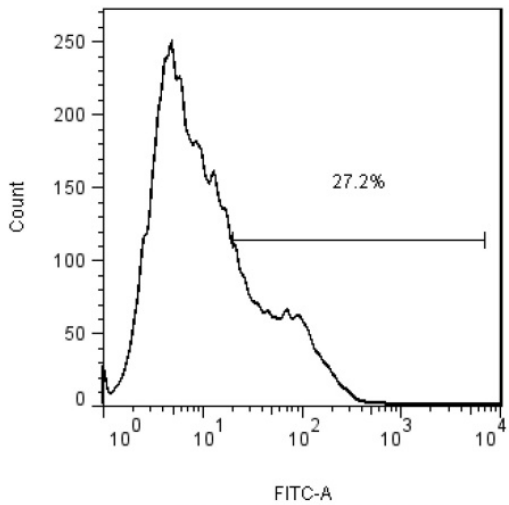

SL 7207 under aerobic

Figure 1. The in vitro flow cytometry results of the annexin $\mathrm{V}$ assay after the co-culture with the 2 strains of Salmonella under the anaerobic and aerobic conditions. YB1 induced 3 folds higher apoptosis in anaerobic than in aerobic condition (31.4\% vs $10.9 \%)$. Such differential effect was not noted in SL7207 treated cells. In addition, the differential killing effect between YB1 and SL7202 under anaerobic condition was not significantly different in the in vitro short term culture setting. 

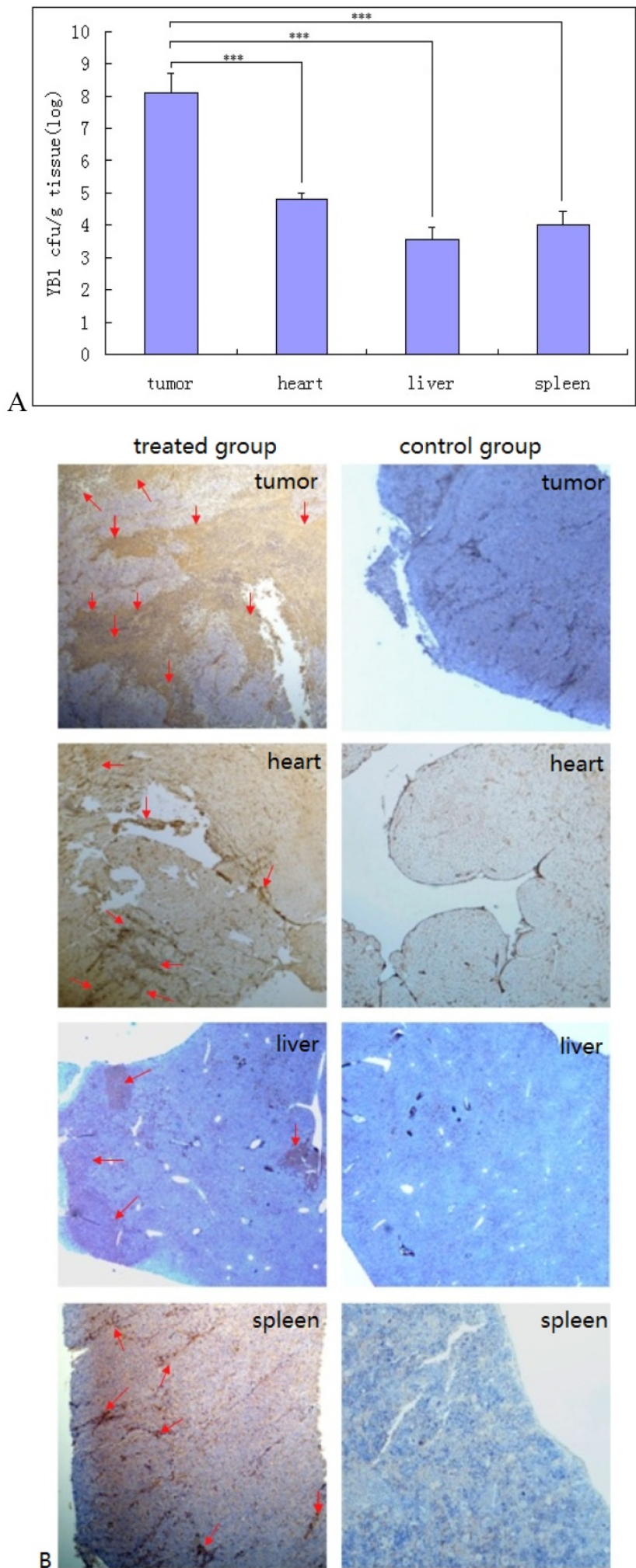

Figure 2. Accumulation of $\mathrm{YBI}$ in tumor and normal tissues in vivo. $A$, Preferential accumulation of $\mathrm{YBI}$ in the orthotopic tumors from mice administered systemically. SCID/beige mice bearing orthotopic SK-NLP/luciferase tumors were injected through tail vein with YB1 $\left(5 \times 10^{7}\right.$ CFU) at day 14. The CFU amounts of accumulated $Y B I$ (means $\pm S D, n=6$ ) in the tumors, hearts, livers, and spleens were determined at day 28 . $* * * P<0.01$. $B$, The immunohistochemical staining analysis of $\mathrm{YBI}$ distribution. After 2 weeks' treatment with YB1, SCID/beige mice were euthanized and YB1 accumulation in tumors, hearts, livers and spleens were assessed in tissue sections by immuno-staining (brown color pointed with red arrow). The left panel was YBI treated group, the right panel was control group.

\section{Observation of the side-effects}

The mice inoculated with $5 \times 10^{7}$ SL7207 started to die on day 3 . On day 8 , all mice were killed because of infection. The CFU from the feces was $2.98 \pm 2.15 \times 10^{5} / \mathrm{g}(\mathrm{n}=6)$, and negative from urine in SL7207 treated mice. However, the mice injected with YB1 were all alive and active till to the end point of the experiment, and there was no bacteria detected either from feces or from urine. For the YB1 treated group, it showed that there was significant initial body weight loss when compared to the non-bacterial treated control ( $p$ values were shown in Fig.3). This might be due to some underlying toxic effect of the bacteria, especially in the early phase. The body weight started to recover after 6 days treatment (Fig. 3).

\section{YB1 inhibited neuroblastoma growth in vivo}

As YB1 invaded SK-NLP/luciferase neuroblastoma cells in vitro causing cell apoptosis, its in vivo therapeutic effect was evaluated. Tumor sizes and growth were monitored by using a Xongen IVIS 100. The data showed that tumor growth in YB1 treated mice was significantly retarded compared with PBS treated mice ( $p=0.0094$ ) (Fig. $4 A, B, C$ and $D)$. No antitumor effect could be detected at Day 14 in SL7207 treated mice since all mice died between day 3 and 8.

\section{The expression of TLR4 and 5 under the invasion of YB1 and SL7207}

Quantitative-PCR revealed that all TLR4 and 5 were up-regulated after bacterial invasion. TLR4 gene expression was significantly higher after treatment with YB1 and SL7207 under anaerobic conditions than under aerobic conditions. The $p$ values were 0.017 and 0.041 respectively and the $p$ values of TLR5 gene were $p=0.001$ and $p=0.04$ respectively (Fig. 5A). The experiment was performed in triplicate. In the tumor mass, the expression of TLR4 and 5 were also higher in YB1 treated group than control group from the results of the immunohistochemical staining (Fig. 5B, $C, D$ and $E$ ).

\section{Discussion}

Neuroblastoma derived from aberrant sympathetic nervous system development. Due to the differences in the underlying genetic involvement, it exhibits diverse clinical behaviors. Some may spontaneously regress, while the aggressive forms in older children still account for a significant proportion of morbidity and mortality in children with cancers. Neuroblastoma represents for around $8 \%$ to $10 \%$ of all childhood cancers but takes up approximately $15 \%$ share of cancer deaths in children [16]. Advanced 
stage tumors often infiltrate local organ structures, wrapping around nerves and vessels and are unresectable with extremely poor outcome [17-19]. Despite recent advances on diagnosis and therapy, $50 \%$ to $60 \%$ of patients with high-risk neuroblastoma relapse, and to date there are no curative salvage treatment regimens available [20]. Current treatment for neuroblastoma included surgical resection, chemotherapy, radiotherapy, and biologic therapy such as isotretinoin (cis-RA), anti-GD2 immunotherapy (3F8 or Ch14.18 and Ch14.18/CHO) [21-26].

To be able to sustain continuous growth, solid tumors need to induce neovascularization to ensure sufficient supply of oxygen and nutrients. However, tumor-induced vessels are often malformed, resulting in inadequate blood circulation and accompanying hypoxia [27, 28]. Hypoxia provokes a general adaptive response in neuroblastoma cells and those survived show loss of the neuronal phenotype, gains of stem-cell characteristics such as self-renewal and tumor propagation in vivo.

This cell subpopulation often resides in the core of the solid tumor, which is hypoxic, and was found to have the characteristics of cancer stem cells. Hypoxia promotes expression of genes that mediate cell survival, growth, and resistance to treatment. The genes included HIF-1a, HIF-2a, glucose transporter 3 (GLUT3), vascular endothelial growth factor (VEGF), IGF-1, IGF-2, insulin receptors, neuropilin-1 (NRP1), metallothioneins, MT2A and MT1H et al. [4]. All these make these special neuroblastoma cells adapted to hypoxic environment more resistant to therapy since they have a lower proliferation rate; are exposed to lower concentration of cytotoxic drugs which rely on delivery via the circulation; and are less sensitive to radiation therapy due to the resistant to oxidative damage.

In order to mimic the generation and microenvironment of human neuroblastoma, we utilized the fluorescent orthotopic xenograft mouse model of human neuroblastoma. The tumor was generated by direct adrenal injection, because more than $80 \%$ of human neuroblastoma arises from adrenal gland and adrenal injection can mimic the microenvironment of neuroblastoma [3]. This animal model can be directly visualized with the expressed gene of luciferase in the SK-NLP cells, and is a non-invasive and reliable way to assess the tumor progression and metastatic potential of neuroblastoma. In this model, we were able to achieve $100 \%$ tumor engraftment rate in the SCID/beige mice, and they could all be visually monitored under the Xenogen IVIS 100.

Genetic modified anaerobic bacteria provide an alternative treatment strategy in cancer therapy due to their ability to target the hypoxic core of solid tumors that is resistant to conventional treatment $[29,30]$. If Salmonella, a facultative anaerobic bacterium, is to be a successful treatment agent in anti-cancer therapy, then the potential problems with its toxicity to the host need to be resolved [8, 31]. In most cases attenuated forms are created and used as test therapeutic agents [9, 32-34]. However the mutations required attenuating a bacterium might also compromise its tumor targeting and killing ability. Recently, a systematic study of Salmonella mutants [35] partially resolved this issue by identifying several attenuated mutant bacteria with either mild or moderate reductions in tumor fitness.

Body Weight Loss Percentage

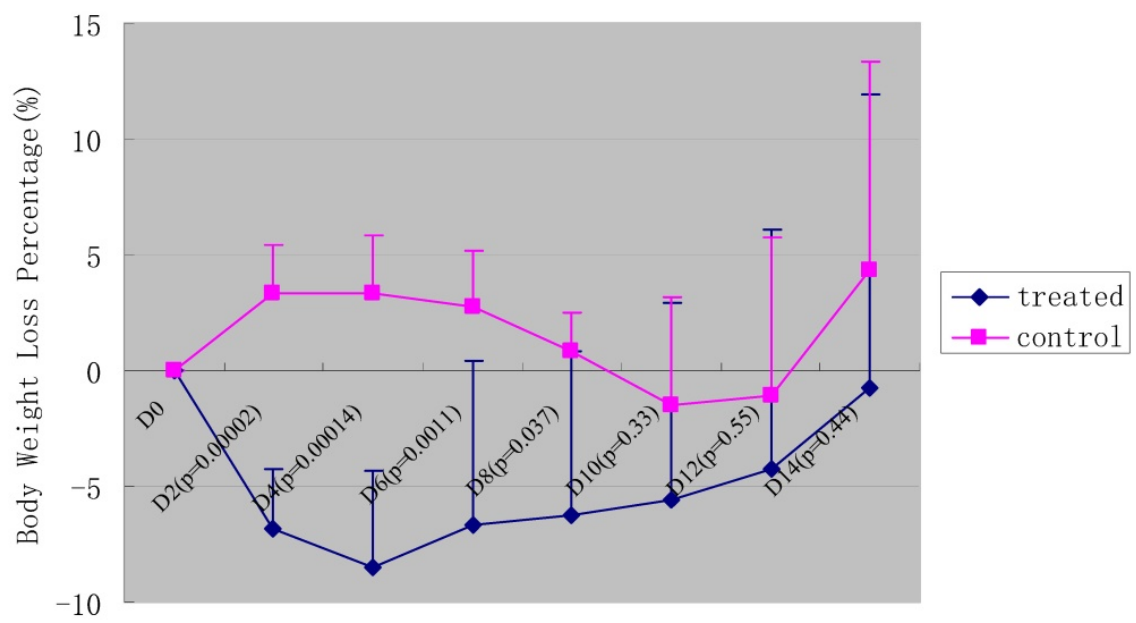

Figure 3. The percentage of body weight loss of the mice after the injection of YBI and PBS. The green line panels belonged to the YBI treated group, and the pink line panels belonged to the control group. The body weight was observed every two days. In general, the body weight loss among the YBI treated group was more than that of the control group during the initial 8 days after the YBI injection (each $p<0.05$ ), especially during the initial 6 days (each $p<0.01$ ). The $p$ values of each time point were showed above. 

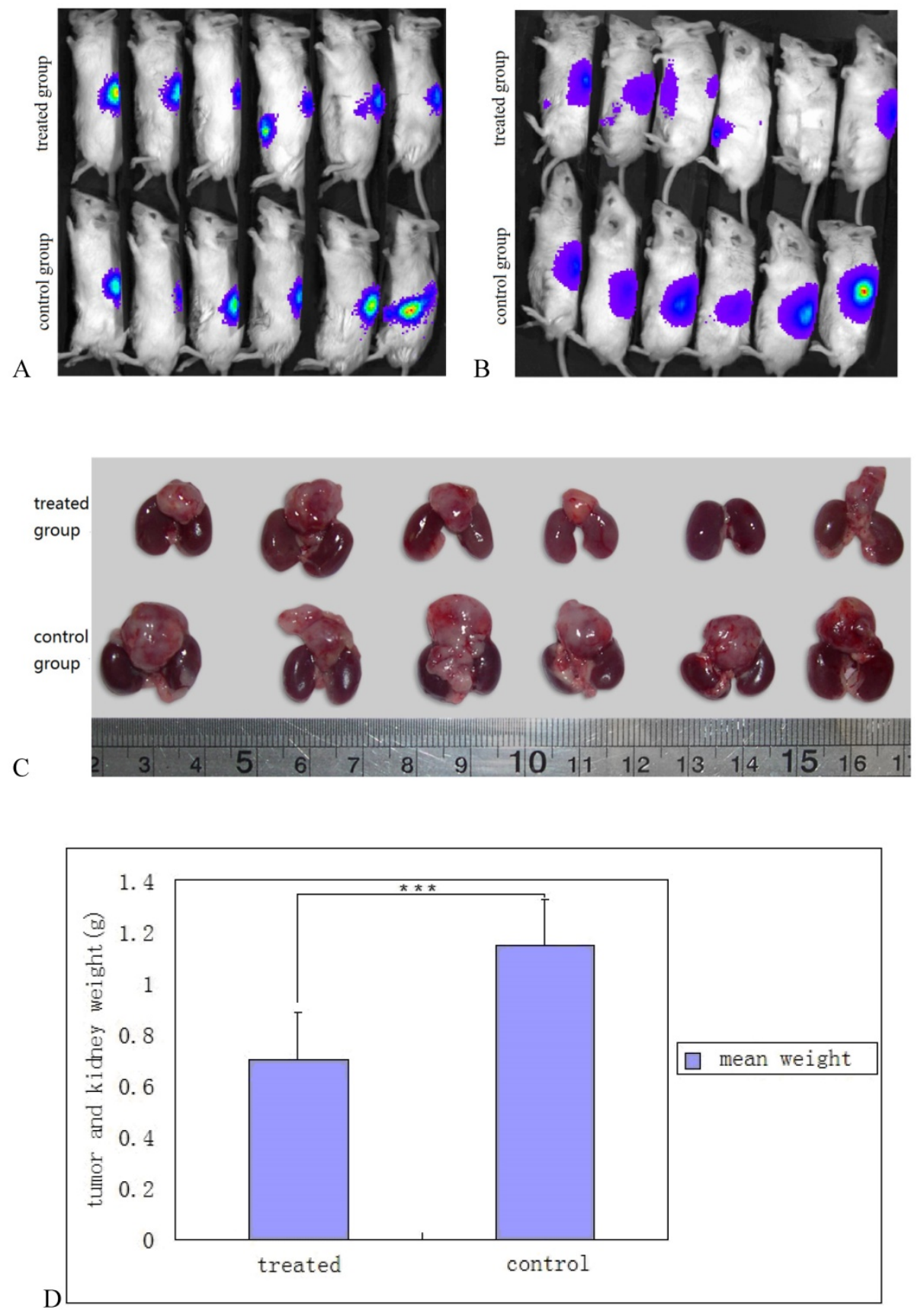

Figure 4. Salmonella YB1 inhibited neuroblastoma growth in vivo. $A$ and $B$, monitoring of in situ neuroblastoma growth by Xenogen IVIS 100 after SK-NLP/luciferase cells implantation and $\mathrm{YBI}$ treatment. Fig. $5 A$ was taken after 2 weeks of neuroblastoma implantation and before the bacterial inoculation. The upper panel was assigned to $Y B 1$ treatment and the lower panel represented the control group. Fig. 5B was taken 2 weeks after $Y B 1$ and PBS injection. The intensity of luciferin signals significantly decreased, not only at the primary tumor but also at the metastatic sites. For treated mouse 5 , there was no more luciferin signal detected; $C$, decrease in tumors size of neuroblastoma after $Y B 1$ treatment. The upper panel was YBI treated group, the lower panel was control group. The tumor is the mass detected on top of the 2 kidneys also shown for comparison. In general, the tumors size of the YBI treated group was significantly smaller than that of control group. The tumor mass resolved completely in mouse $5 ; D$, the mean values of tumor and kidney weight between the YBI treated and control groups. There was statistically significant less tumor weight in the YBI treated group when compared to the control group. The data were expressed as mean \pm SD. $* * * P<0.01$.

Instead of attempting to attenuate the potency of the Salmonella bacteria, we utilized an alternative approach in using genetic modified Salmonella YB1 which make them not viable in normal tissues with normal oxygen gradient. YB1 was designed to survive and grow only under anaerobic condition to target hypoxic regions of solid tumor. Previously, we showed that YB1 can retard the growth of breast cancer by using nude mice model [8]. In this study, we demonstrated that YB1 can also retard the growth of neuroblastoma carried by SCID mice in addition to nude mouse breast cancer model tested before. The SCID mice have no functional B cells and T cells and are more immune-deficient than nude mice which have no functional $\mathrm{T}$ cells. However, YB1 still accumulated inside tumor and caused the tumor 
regression in both neuroblastoma SCID/beige mice model and nude mice model [15] without killing the treated mice. This result not only further proved the success engineering of YB1 strain, but also indicated the innate immune response played an important role in Salmonella cancer therapy, which was demonstrated by our previous publication [15].

The TLRs are transmembrane proteins expressed by cells of the innate immune system, which recognize invading microbes and activate signaling pathways that launch immune and inflammatory responses. Recent research shows TLRs are expressed on a series of tumor cells, such as colon cancer, breast cancer, prostate cancer, melanoma, lung cancer and ovarian tumors [36,37], and some of them may act as a double-edged sword, enhancing host immunity against the tumor by stimulating antigen presenting cells, and protecting the tumor from host surveillance [38-40].
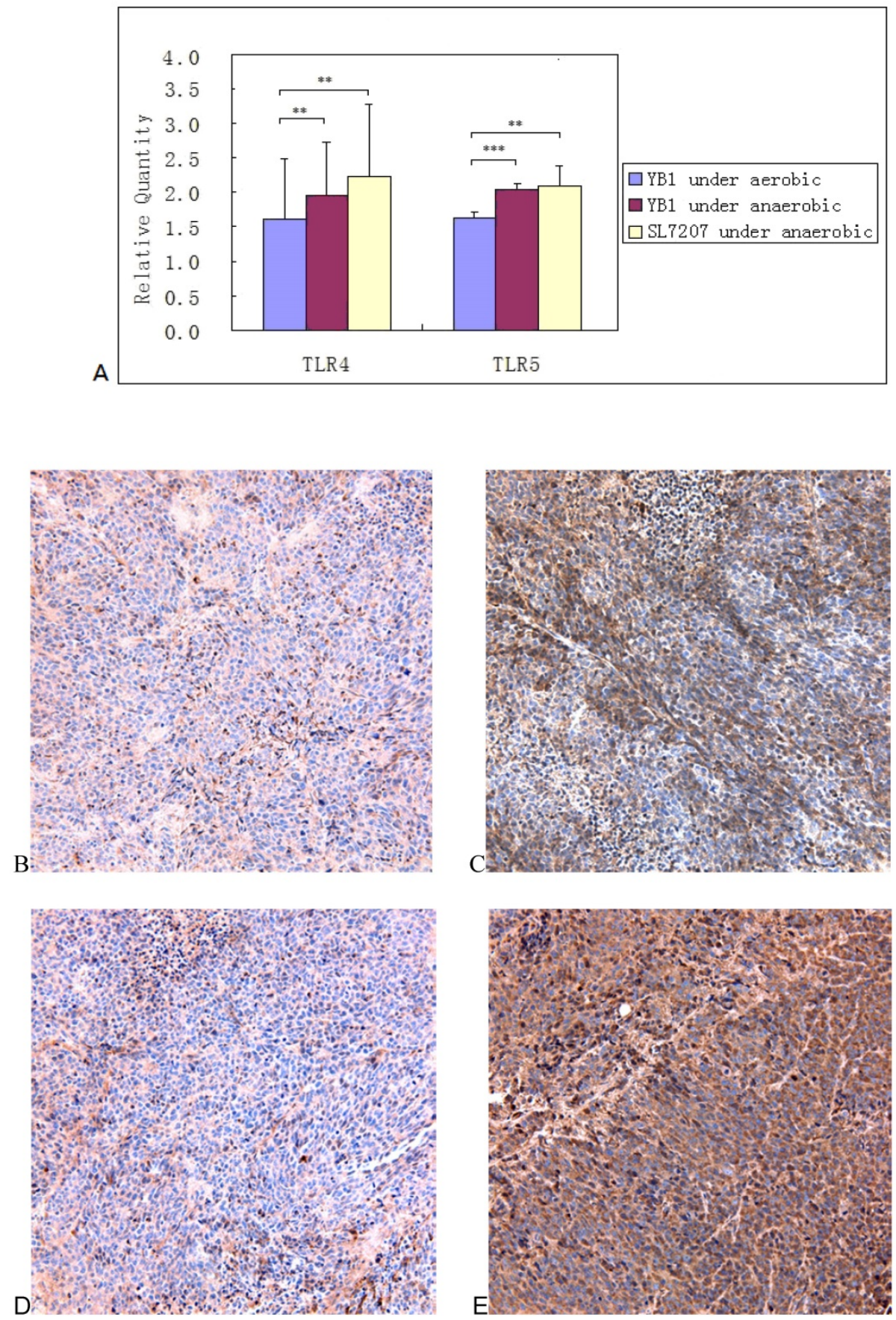

Figure 5. The expression of TLR4 and 5 under the invasion of YB1 and SL7207. A, TLR4 and TLR5 expression of neuroblastoma cells increased under anaerobic condition. The expression of TLR-4,5 genes on SK-NLP/luciferase cells after treated with YB1 and SL7207 under anaerobic and YB1 under aerobic conditions. The data were pooled from the experiments done in triplicate, and expressed as mean \pm SD. $* * P<0.05 ; * * * P<0.01 ; B-E$, the ICH results from the TLR4 and 5 expression in the tumor. TLR4 stain: $B$ and $C$; TLR 5 stain: $D$ and $E$; YB1 treated tumor: $C$ and $E$; control: $B$ and $D$. 
Since the Salmonella typhimurium is gram negative bacteria with abundant LPS and flagellin, and TLR4 and 5 are the receptors of LPS and flagellin, respectively, we performed the TLR4 and 5 genes detect through the RT-PCR after the treatment of YB1. The Quantitative-PCR showed that TLR4 and 5 genes expressed on the SK-NLP cells, for TLR4 and 5 genes expression, there were significant difference between the group of YB1 treated under aerobic conditions and the group of $\mathrm{YB} 1$ treated under anaerobic condition $(P<0.05, P<0.05)$ (Fig. $5 A)$. The same results between the group of $\mathrm{YB} 1$ treated under normoxia and the group of SL 7207 treated under hypoxia $(P<0.01$, $P<0.05)$. The result was consistent with the TLR 4 and 5 protein expression in vivo (Fig. $5 B, C, D$ and $E$ ). These results might indicate that only alive bacteria could induce strong innate response though TLR4 and 5 , but the dead bacteria had little effect.

In vitro, YB1 induced about $31.4 \%$ SK-NLP cells apoptosis, almost the same efficacy as SL7207 (29.1\%), and it is only $10.8 \%$ under normoxia (Fig. 1). This result further proved that YB1 has been successfully converted to an "obligate" anaerobe with removing the lethal toxicity of the host strain while maintaining its tumor invasion under hypoxia [8].

In vivo, YB1 showed strong infiltration into neuroblastoma in SCID/beige mice model, as evidenced by the significant count of bacteria found in the tumors over normal organs $(P<0.01)$ and the tumors weight compared to the PBS control group $(P<0.01)$. Very few YB1 cells were found in the normal tissues as hearts, spleens and livers, and there were no bacteria detected in brains, lungs, kidneys and blood after two weeks of YB1 treatment (Fig. 2). This is in sharp contrast with SL7207 treated mice where bacterial cells distributed with high CFU in normal organs, which caused the mice dead rapidly. When compared with untreated mice, YB1 considerably retarded tumor growth without affecting the survival of the animals except for the initial body weight loss (Fig. 3, Fig. 4A, 4B, $4 C$ and $4 D$ ).

As the expansion of the previous study [15], this study further demonstrated the direct killing effect of Salmonella under both in vitro and in vivo setting. We also analyzed the dynamic side-effects profile of YB1 through survival rate, body weight loss over time, tissue distribution of bacteria, etc. These further verified the potential clinical application of YB1 in solid tumors.

In conclusion, we have demonstrated that systemic administration of tumor targeting YB1 induced apoptosis on neuroblastoma cells and resulted in tumor growth suppression. While the bacteria targeted at the cancer cells in the tumor core, it did not induce significant damage or dysfunction of other normal organs or tissues, as our previous report [8]. Therefore, our data suggested YB1 could be a useful tool in delivering anti-neuroblastoma agents. It has the potential of targeting the cancer cells at the hypoxic core of the tumors with a totally different cytotoxic mechanism comparing to conventional chemotherapeutic agents. These results also indicated that YB1 as an anticancer agent may not only specific to some cancer, but also could be a common weapon against many solid tumors. Further work is warranted to elucidate the underlying mechanism of tumor target killing effect of the genetically engineered anaerobic YB1. The effect of such approach in immune-competent mice may also warrant further exploration.

\section{Acknowledgments}

This work was supported by CRF Grant from the RGC (HKU1/CRF/10) to JDH. BTN was supported by a Dr Cheng Yu Tung Fellowship and the project of Zhejiang Provincial Natural Science Foundation of China (LY16H160024).

\section{Competing Interests}

The authors have declared that no competing interest exists.

\section{References}

1. London WB, Castleberry RP, Matthay KK, Look AT, Seeger RC, Shimada H, et al. Evidence for an age cutoff greater than 365 days for neuroblastoma risk group stratification in the Children's Oncology Group. Journal of clinical oncology : official journal of the American Society of Clinical Oncology. 2005; 23: 6459-65.

2. Vitali R, Mancini C, Cesi V, Tanno B, Piscitelli M, Mancuso M, et al. Activity of tyrosine kinase inhibitor Dasatinib in neuroblastoma cells in vitro and in orthotopic mouse model. International journal of cancer Journal international du cancer. 2009; 125: 2547-55.

3. Henriksson KC, Almgren MA, Thurlow R, Varki NM, Chang CL. A fluorescent orthotopic mouse model for reliable measurement and genetic modulation of human neuroblastoma metastasis. Clinical \& experimental metastasis. 2004; 21: 563-70.

4. Jogi A, Vallon-Christersson J, Holmquist L, Axelson H, Borg A, Pahlman S. Human neuroblastoma cells exposed to hypoxia: induction of genes associated with growth, survival, and aggressive behavior. Experimental cell research. 2004; 295: 469-87.

5. Brown JM, Giaccia AJ. The unique physiology of solid tumors: opportunities (and problems) for cancer therapy. Cancer research. 1998; 58: 1408-16.

6. Wei MQ, Ellem KA, Dunn P, West MJ, Bai CX, Vogelstein B. Facultative or obligate anaerobic bacteria have the potential for multimodality therapy of solid tumours. European journal of cancer. 2007; 43: 490-6.

7. Pawelek JM, Low KB, Bermudes D. Tumor-targeted Salmonella as a novel anticancer vector. Cancer research. 1997; 57: 4537-44.

8. Yu B, Yang M, Shi L, Yao Y, Jiang Q, Li X, et al. Explicit hypoxia targeting with tumor suppression by creating an "obligate" anaerobic Salmonella Typhimurium strain. Scientific reports. 2012; 2: 436.

9. Forbes NS, Munn LL, Fukumura D, Jain RK. Sparse initial entrapment of systemically injected Salmonella typhimurium leads to heterogeneous accumulation within tumors. Cancer research. 2003; 63: 5188-93.

10. Leschner S, Westphal K, Dietrich N, Viegas N, Jablonska J, Lyszkiewicz M, et al. Tumor invasion of Salmonella enterica serovar Typhimurium is accompanied by strong hemorrhage promoted by TNF-alpha. PLoS One. 2009; 4:

11. Loessner H, Endmann A, Leschner S, Westphal K, Rohde M, Miloud T, et al. Remote control of tumour-targeted Salmonella enterica serovar Typhimurium by the use of L-arabinose as inducer of bacterial gene expression in vivo. Cell Microbiol. 2007; 9: 1529-37.

12. Royo JL, Becker PD, Camacho EM, Cebolla A, Link C, Santero E, et al. In vivo gene regulation in Salmonella spp. by a salicylate-dependent control circuit. Nat Methods. 2007; 4: 937-42. 
13. Westphal K, Leschner S, Jablonska J, Loessner H, Weiss S. Containment of tumor-colonizing bacteria by host neutrophils. Cancer research. 2008; 68: 2952-60.

14. Hoiseth SK, Stocker BA. Aromatic-dependent Salmonella typhimurium are non-virulent and effective as live vaccines. Nature. 1981; 291: 238-9.

15. Guo ZL, Yu B, Ning BT, Chan S, Lin QB, Li JC, et al. Genetically modified "obligate" anaerobic Salmonella typhimurium as a therapeutic strategy for neuroblastoma. Journal of hematology \& oncology. 2015; 8: 99.

16. Park JR, Eggert A, Caron H. Neuroblastoma: biology, prognosis, and treatment. Hematology/oncology clinics of North America. 2010; 24: 65-86.

17. Carlsen NL. How frequent is spontaneous remission of neuroblastomas? Implications for screening. British journal of cancer. 1990; 61: 441-6.

18. Cole WH, Everson TC. Spontaneous regression of cancer: preliminary report. Annals of surgery. 1956; 144: 366-83.

19. Yamamoto K, Hanada R, Kikuchi A, Ichikawa M, Aihara T, Oguma E, et al. Spontaneous regression of localized neuroblastoma detected by mass screening. Journal of clinical oncology : official journal of the American Society of Clinical Oncology. 1998; 16: 1265-9.

20. Maris JM. Recent advances in neuroblastoma. The New England journal of medicine. 2010; 362: 2202-11.

21. Barker E, Mueller BM, Handgretinger R, Herter M, Yu AL, Reisfeld RA. Effect of a chimeric anti-ganglioside GD2 antibody on cell-mediated lysis of human neuroblastoma cells. Cancer research. 1991; 51: 144-9.

22. Barker E, Reisfeld RA. A mechanism for neutrophil-mediated lysis of human neuroblastoma cells. Cancer research. 1993; 53: 362-7.

23. Hank JA, Robinson RR, Surfus J, Mueller BM, Reisfeld RA, Cheung NK, et al. Augmentation of antibody dependent cell mediated cytotoxicity following in vivo therapy with recombinant interleukin 2 . Cancer research. 1990; 50: 5234-9.

24. Yu AL, Gilman AL, Ozkaynak MF, London WB, Kreissman SG, Chen HX, et al. Anti-GD2 antibody with GM-CSF, interleukin-2, and isotretinoin for neuroblastoma. The New England journal of medicine. 2010; 363: 1324-34.

25. Osenga KL, Hank JA, Albertini MR, Gan J, Sternberg AG, Eickhoff J, et al. A phase I clinical trial of the hu14.18-IL2 (EMD 273063) as a treatment for children with refractory or recurrent neuroblastoma and melanoma: a study of the Children's Oncology Group. Clinical cancer research : an official journal of the American Association for Cancer Research. 2006; 12: 1750-9.

26. George RE, Diller L, Bernstein ML. Pharmacotherapy of neuroblastoma. Expert opinion on pharmacotherapy. 2010; 11: 1467-78.

27. Brown JM. Exploiting the hypoxic cancer cell: mechanisms and therapeutic strategies. Molecular medicine today. 2000; 6: 157-62.

28. Vaupel P, Thews $\mathrm{O}$, Hoeckel M. Treatment resistance of solid tumors: role of hypoxia and anemia. Med Oncol. 2001; 18: 243-59.

29. Brown JM, Wilson WR. Exploiting tumour hypoxia in cancer treatment. Nat Rev Cancer. 2004; 4: 437-47.

30. Pawelek JM, Low KB, Bermudes D. Bacteria as tumour-targeting vectors. The lancet oncology. 2003; 4: 548-56.

31. Leschner S, Weiss S. Salmonella-allies in the fight against cancer. J Mol Med (Berl). 2010; 88: 763-73

32. Clairmont $\mathrm{C}$, Lee $\mathrm{KC}$, Pike J, Ittensohn $\mathrm{M}$, Low KB, Pawelek J, et al. Biodistribution and genetic stability of the novel antitumor agent VNP20009, a genetically modified strain of Salmonella typhimurium. J Infect Dis. 2000; 181: 1996-2002.

33. Song M, Kim HJ, Kim EY, Shin M, Lee HC, Hong Y, et al. ppGpp-dependent stationary phase induction of genes on Salmonella pathogenicity island 1. J Biol Chem. 2004; 279: 34183-90.

34. Zhao M, Yang M, Ma H, Li X, Tan X, Li S, et al. Targeted therapy with a Salmonella typhimurium leucine-arginine auxotroph cures orthotopic human breast tumors in nude mice. Cancer research. 2006; 66: 7647-52.

35. Arrach N, Cheng P, Zhao M, Santiviago CA, Hoffman RM, McClelland M. High-throughput screening for salmonella avirulent mutants that retain targeting of solid tumors. Cancer research. 2010; 70: 2165-70.

36. Huang B, Zhao J, Li H, He KL, Chen Y, Chen SH, et al. Toll-like receptors on tumor cells facilitate evasion of immune surveillance. Cancer research. 2005; 65: 5009-14.

37. Zhou M, McFarland-Mancini MM, Funk HM, Husseinzadeh N, Mounajjed T, Drew AF. Toll-like receptor expression in normal ovary and ovarian tumors. Cancer immunology, immunotherapy : CII. 2009; 58: 1375-85.

38. Szczepanski MJ, Czystowska M, Szajnik M, Harasymczuk M, Boyiadzis M, Kruk-Zagajewska A, et al. Triggering of Toll-like receptor 4 expressed on human head and neck squamous cell carcinoma promotes tumor development and protects the tumor from immune attack. Cancer research. 2009; 69: 3105-13.

39. Vulcano M, Dusi S, Lissandrini D, Badolato R, Mazzi P, Riboldi E, et al. Toll receptor-mediated regulation of NADPH oxidase in human dendritic cells. J Immunol. 2004; 173: 5749-56.

40. Woods DC, White YA, Dau C, Johnson AL. TLR4 activates NF-kappaB in human ovarian granulosa tumor cells. Biochemical and biophysical research communications. 2011; 409: 675-80. 